\title{
Cultivating the Sense-of-the-Other/Sense of Community: An Autoethnographic Case Study of Psychotherapy with High-Risk, Urban Adolescents
}

\author{
Gen Nakao \\ Department of Management, Otemon Gakuin University, Osaka, Japan \\ Email: g-nakao@otemon.ac.jp, gennakao@gmail.com
}

How to cite this paper: Nakao, G. (2021). Cultivating the Sense-of-the-Other/Sense of Community: An Autoethnographic Case Stu-dy of Psychotherapy with High-Risk, Urban Adolescents. Current Urban Studies, 9, 196-205.

https://doi.org/10.4236/cus.2021.92012

Received: April 24, 2021

Accepted: May 25, 2021

Published: May 28, 2021

Copyright (C) 2021 by author(s) and Scientific Research Publishing Inc. This work is licensed under the Creative Commons Attribution International License (CC BY 4.0).

http://creativecommons.org/licenses/by/4.0/

\begin{abstract}
This paper presents an autoethnographic case study in order to discuss interpersonal psychotherapy and work conducted with high-risk adolescents in an urban school setting. The author discusses how the sense-of-the-other developed by counseling sessions possibly fosters a sense of connection for students, and the sense of belonging to a school. This paper argues that counselors often encounter students' resistance and/or hesitance to mental health counseling because of a lack of awareness of such services within the wider community. Such cultural barriers should be taken into account in order to understand better the lived-experiences of a community. Group sessions, or the facets of the interactions at school that successfully facilitated self-expression, could function as a safe refuge for students and ultimately develop a sense of community. Such an educational context possibly constitutes a link between marginalized individuals and a school in a community.
\end{abstract}

\section{Keywords}

Sense of Community, Community Building, Autoethnography,

Community-Based Participatory Research, Interpersonal Psychotherapy,

Group Counseling, Urban Education, Counselor Education, New York City

\section{Background}

The application of interpersonal psychotherapy (Levenson, 2005; Sullivan, 1953) to minority students living in low socio-economic communities can be of interest to mental health professionals working with a diverse global population. For instance, children of disaster victims, foreign nationals attending local schools, 
children from families on welfare programs, and children from broken homes are potential candidates who may benefit from such initiatives. In this article, the author reports an autoethnographic case study of school counseling programs in New York City, the United States. Specifically, group counseling exemplifies how an educational context possibly constitutes a link (i.e., linking. Dalton, Elias, \& Wandersman, 2001) between marginalized individuals and a school in a community. Such a link also has the potential to foster a sense of community or place attachment for school members.

\section{The Framework of Interpersonal Psychotherapy}

Interpersonal psychotherapy focuses on patients' symptoms and their current interpersonal context, regardless of the etiology of the disorder (Kazdin \& Weisz, 2003). This approach does not view the client's issues based upon essentialism, nor does it pathologize the clients. The original theorists of this school, Harry Stack Sullivan and Adolf Meyer, shifted the focus of psychiatry away from the individual, contending that one's personality is the culmination of recurrent patterns of interpersonal interactions (Sullivan, 1953). Diagnosis and intervention must identify not only the symptoms and behaviors associated with the disorder, but also examine the (maladaptive) interpersonal interactions and the quality of communication. For instance, Sullivan argued that therapists can translate depression into the underlying interpersonal features, such as a tendency toward passivity, isolation, obsequiousness, inability to express anger, and hypersensitivity to separation (Sullivan, 1953). Incidentally, this interpersonal theory or Neo-Freudian approach (Walrond-Skinner, 1986) resonates with the social constructionist approach, which emphasizes the power of social interaction in generating meaning (Gergen, 1985). According to Hess (2009), the core of the interpersonal approach is the sense-of-the-other, which involves the engagement of a person in the other's life. As described later in this article in relation to group programs, it is noteworthy that students may benefit from contact with school faculties through therapeutic interactions despite low attendance.

\section{Autoethnography as Method: The Case and Reflexive Process in the Cultural Context}

As a methodological practice of postpositivist qualitative inquiries (Gannon, 2017), the author adopted the use of autoethnography (Ellis, Adams, \& Bochner, 2011) to further discuss the lived-experiences of the group sessions. Autoethnography taps into the self-observational and reflexive data of personal experiences, providing in-depth analysis of activities, feelings, and emotions within particular contexts (Grbich, 2012). Given the nature of the group sessions below, this autoethnographic approach is applicable, because the author was a mental health clinician and thus received supervision on a weekly basis, as well as keeping progress notes (i.e., a document recording a client's status and achievements during the course of treatment, designed to be shared with other professionals) 
and reflective notes (i.e., subjective notes for personal use as part of the life-long learning process), all of which contributed to in-depth self-reflection. Also, the autoethnographic approach is viable, as the author is both an insider (being a mental health counselor at the site) and an outsider (being a social/cultural psychologist), holding the perspectives of both. Finally, autoethnography is a valid method in this context because it highlights alterity, or the experience of being different. As a foreign-born therapist working in the United States, the author thus occupies the position of "other" (i.e., experiencing othering: Grbich, 2012).

\section{The Case Study: A Charter School Located in the South Bronx, New York City}

This article provides a case study of working with high-risk adolescents in an urban school setting. Given a pseudonym to ensure anonymity, WWW school is a charter school located in the South Bronx in New York City, which weekly holds two group counseling projects (the XXX project and the YYY project, also anonymized), piloted by a medical school. As described later, the model (see Figure 1) was based on the author's experience of working in the charter school as a mental health counselor intern, summarized in more detail in the autoethnographic section. In that autoethnographic section, the author turns to first-person voice because it highlights the author's lived-experiences in each situation. In this sense, this paper aims to synthesize and propose a hypothesis about what it is like working in an urban school as a (foreign-born) mental health clinician.

\subsection{The School Demographic and the Geographical Setting}

The majority of students at WWW school are African American or Latino teens who reside in a very isolated and economically disadvantaged community in the Hunt's Point area of the South Bronx, one that is known to be a crime-ridden, drug-active, and noted gang-involved neighborhood. WWW school was established as a charter school in 2000 as a last-ditch attempt to serve about 300 students who are over-aged and under-credited so they can earn their high school diplomas. This school's mission is to prepare students with job training skills that match their academic endeavors and thus create a real-world emphasis and training ground for students. Of relevance to this current study, the students have no clear (or formal) membership to the school, negatively affecting a sense of belonging; there are no school newspapers and no after-school clubs. Instead, the students convey a stronger sense of identity and resilience with the many disadvantages in their lives, presenting a take charge presence, even if it involves illegal or risky behaviors that they identify as ways to help them cope with the stress of life. However, informal interactions and relationships between various teachers, support staff, and students possibly enhance the sense of belonging to the school for the students. For instance, some students intentionally drop classes because they do not want to leave the campus. As discussed later, this sense of belonging that students attach to the school, which is not necessarily a 
formal/traditional school model, is strongly related to this author's rationale of utilizing an interpersonal approach.

In terms of student attendance, WWW school has less than a 70\% attendance rate, which has affected clinicians' ability to engage regularly and maintain a working relationship with the teens. When the author discussed this issue with some of the teachers, one stated, "the students here are almost part-timers," acknowledging that it is hard to keep the students at class regularly. Nevertheless, the faculty member and the author recognized the underlying factors, especially that the teens need to work to contribute to household expenses. Also, many teens are from family environments of violence, substance abuse, incarceration, housing instability, the Administration for Children's Services' (ACS) custodial involvement, and neighborhood discord, and some are on out-of-home placements on undocumented status.

\subsection{Subcultural Considerations: Street Codes}

There are a multitude of complex issues serving as barriers to student engagement in mental health treatment, most notably the community's negative experience, perception, and adherence to the stigmas surrounding mental health issues. For most of these students, education is unfortunately not a priority, understandable given everything else they have to deal with on an on-going economic basis. Counselors often encounter resistance to educational norms from the community because community member are not socialized with mental health services, academic values, success, and goals; education is not a part of their street code (Mateu-Gelabert \& Lune, 2007). Such cultural barriers (Vontress, 1969, 1974; Yancy, 1984) must be taken into consideration in order to optimize understanding of the community.

\subsection{Autoethnographic Note (1): My Experience of the School Setting}

As an Asian male originally from Japan, certain barriers arose. Firstly, blending in was not straightforward; I was hesitant to reach out to the students. In relation to the race and power balance between teachers and students, being an Asian male employee positioned me as a minority in terms of race, yet simultaneously as part of the authoritative body (faculty of the school), prompting a duality in relation to how I was perceived and what was expected in my role. This experience made me realize that I was not really aware of racial issues before joining the school. I became interested in how students viewed the faculty members within the community, because there are also African-American and Latino teachers at the school. My ethnicity drew many stares from students in the cafeteria, which I eventually realized were influenced by curiosity. However, the extent of the scrutiny fatigued me and required courage to overcome.

An additional barrier related to how students responded to me as a speaker of English as an additional language. While conversations in the hallway were 
conducted with ease, in the classroom I was not always able to make myself understood clearly, such as in group counseling sessions as well as when observing and overseeing a classroom. This was probably because classroom English tends to be more formal, whereas hallway conversations were conducted in a vernacular closer to the street code of the community (Mateu-Gelabert \& Lune, 2007), where my informal and conversational English was accepted.

Outside formal counseling sessions, I was able to familiarize myself with the school because the students engaged with me on a catch-as-catch-can basis, in the hallways or outside of class, and sometimes in the counseling office. Observing classes was particularly effective because it allowed students to recognize me outside the counseling sessions, and the students and I could share topics taught during classes and thus the context of the school.

\subsection{The Content and Nature of Group Counseling Sessions: XXX and YYY Projects}

As mentioned before, the author facilitated two kinds of group counseling sessions with his supervisor, one of which was the XXX project that focused on reproductive health care and healthy relationships, and the other was the YYY project, which was a positive youth development/mental health intervention.

The XXX project is funded through the NY Community Trust and is a project designed to educate and engage teens in the Bronx to explore the multitude of issues about reproductive health care and developing healthy relationships. This program covers the following topics: sexual relationships, values, gender norms, building healthy relationships, male and female anatomy, pregnancy, HIV/AIDS and STDs, risky sexual behaviors, drug/alcohol abuse, communication skills, and birth control options, including abstinence, barrier methods (such as condom use), and hormonal methods (such as the birth control pill). The information is presented through large and small group discussions, co-educational and separate discussions for male and female students, and role-play situations.

The XXX project explores and promotes awareness and implications of traditional gender norms and the potentially negative impact of risky sexual behaviors. For example, gender norms often dictate that a girl should not carry condoms, which would be interpreted as implying that she is sexually active or even promiscuous, which in this peer group can lead to a bad reputation. However, by adhering to this gender norm, teenage girls are at an increased risk of pregnancy and/or infection. The overall goal of the program is to empower both male and female participants by giving them a repertoire of skills that they can use to make healthier decisions about sex, while navigating their way through complex dilemmas caused by gender norms and sexual relationships.

The YYY project is part of a larger research scheme (community-based participatory research: CBPR) conducted through a medical school research center. Funding for this program is provided by the National Institute on Minority Health and Health Disparities. The YYY project is a positive youth development 
and mental health intervention created by teens for teens, with the aim to reduce mental health disparities among young African American and Latino people in the Bronx. Topics include communication skills, problem-solving, conflict resolution, time management, goal setting, mental health, dealing with teen issues, and coping with stress, as well as other relevant issues that teens may bring up that relate to mental health. The YYY project is also based on a train-the-trainer model of prevention, coaching these participants to become peer educators in their communities.

\subsection{Autoethnographic Note (2): My Experience of the Group Sessions}

When first leading group sessions, I was at a loss regarding how I should or could successfully work with the population. This was because individual sessions (separate from the group sessions) were not constantly held at the school, and reaching out to the students was therefore difficult. I felt desperate, questioning my value at the school. However, as stated above, understanding the subcultural factors that prompted resistance to therapy was helpful in understanding the population.

Leading these groups, I was shocked and downhearted to see that many of the teens in this community struggled with the following issues: poor attendance (which significantly impaired their academic performance), pre- and post-incarceration and parole issues (whether for themselves as juveniles or within their families), teen pregnancies, intimate partnership abuse, gang violence, ACS custodial involvement, out-of-home placements, undocumented immigrant status, poverty, drug and alcohol abuse, medical concerns, and apathy. With these teenagers in particular, such issues, combined with a notable lack of support in their lives, significantly contribute to high-risk behaviors.

Knowing these issues, I contemplated how the two group programs could effectively relate to this community. In other words, I began wondering about the power relationship between a medical school piloting the groups sessions and the high school that received it. This notion resulted in the following three aspects. First, I came to realize that it was vital not to exploit the community for self-interest. Instead of focusing on producing research publications for personal advancement, clinicians should focus on writing progress notes or case reports on a daily basis. Considering this, I gave careful thought about whether I tend to look for a topic on which to write a report, rather than responding to the clients and problems I encounter in the field.

Second, I became aware of my own privilege. For instance, I realized that my medical school ID-card worked as a signifier or symbol of authority, because it garnered significant attention from students in the hallway. I was surprised that this lanyard conveyed such meaning, and I looked for an appropriate way to respond and reach out to the gazing students, because an authoritative figure for them meant someone who could grade, evaluate, and report misbehavior to oth- 
er teachers. This pejorative association with authority prompted me at one point to reassure students that I was not a teacher's spy, because they were concerned about evaluation. Also, although the group program was an affiliative partnership, it was the medical school that made decisions about it (i.e., ownership of the activities).

Third, I realized that I undoubtedly appreciate the value of higher education. As stated before, access to education was not always prioritized in this community, and there was the risk that I might have overlooked the reasons behind this. Accordingly, beyond my simple emotional reactions, looking back I can see that those three notions on power relations could have raised my awareness of my own value and understanding of the world, which would involve gaining critical consciousness or conscientization (Freire, 2000).

In addition to those reflective processes, I also made three analyses of the groups. First, participants were asked to summarize the sessions and write their reactions after each group session in a journal, and this seemed a helpful activity. For instance, the journals revealed that some students who appeared to be simply staring off into space in class were in fact absorbing a lot of what went on, to the extent that they were able to convey their understanding about the topics in the journals.

Second, I realized that cross-cultural consideration is necessary when working with this population. The concept of being inside or outside (i.e., inside/outside of culture) is important, because on the one hand I needed to know the background of my clients (being in the culture and context), while on the other hand, I should have suspended any preexisting biases or assumptions before conducting sessions with students (being out of the culture and context). This seems contradictory, yet occupying both perspectives is necessary in order to ensure that a counselor is knowledgeable yet non-judgmental about their clients' backgrounds.

Third, the participants seemed to engage more with creative activities such as discussion, acting (role-play), and even rap performances, especially when compared with solely academic activities (i.e., anatomy and STDs). Further, activities or topics that they could relate to their own lives also inspired engagement. For instance, when discussing relationship breakdowns, one male student performed his Spanish rap to express his feelings to the group, which had the added benefit of developing his sense of belonging. Notably, group sessions that successfully implemented such a personal disclosure would help the members to go under the meaning-making process or appropriation, a process in which people take something that belongs to others and make use of it for certain purposes (cf. Bakhtin, 1981; Esteban-Guitart, 2014). Such a co-construction of meaning process might help to develop the motivation for a member to adhere to a group, because mutual self-disclosure would involve the sense of engagement of a person in the other's life, on which interpersonal therapy places much importance.

Accordingly, in the framework of interpersonal psychotherapy, I learned that 
despite the limitations placed on engaging in formal treatment, the students were able to form a trusting bond with counselors through the group sessions. One positive outcome for the students is an increase in the closeness and quality of their relationship or membership with the school, which might enhance attendance and engagement (Anderson, Christenson, Sinclair, \& Lehr, 2004). Conceptually, this resonates well with a psychological sense of community (Sarason, 1974) or place attachment (Hidalgo \& Hernández, 2001), which are widely discussed in community psychology. According to Sarason (1974), the psychological sense of community is defined as:

The perception of similarity to others, an acknowledged interdependence with others, a willingness to maintain this interdependence by giving to or doing for others what one expects from them, and the feeling that one is part of a larger dependable and stable structure (p. 157).

Hence, opportunities to self-disclose in a group session, including through the medium of rap, can develop a sense of community.

As indicated by the example of the Spanish rap performance, group counseling sessions can be a safety spot or safe refuge, one where members can listen to each other. Combining interpersonal psychotherapy, the following diagram can be tailored for school counseling sessions that possibly foster a sense of community.

Stage 1: The sense-of-the-other developned by group (and/or individual) counseling sessions

Stage 2: Tangible connection and engagement with someone at school

Stage 3: Attachment or the sense of belonging based on connection at school

Stage 4: Increased sense of community (e.g., improving attendance at school)

Figure 1. Stage model showing the microcosm of interpersonal relationships at school.

According to the interpersonal psychotherapy model, the sense-of-the-other provided by group counseling can foster an almost tangible sense of connection with someone at school. Such a connection means that by coming to school, students can meet someone (e.g., a faculty member, a school staff member, or a counselor) with whom they can speak, even if casually. Such a sense of engagement in the school can develop a sense of belonging, which in turn can lead to an ultimate sense of community or place attachment. Such a sense of community might also result in improved attendance rates once students feel that they are part of the school system.

\section{Conclusion}

This paper presented an autoethnographic case study in order to discuss interpersonal psychotherapy and work conducted with high-risk adolescents in an urban school setting. The author discussed how the sense-of-the-other developed by counseling sessions possibly fosters a sense of connection for students, 
and the sense of belonging to a school. This paper argued that counselors often encounter students' resistance and/or hesitance to mental health counseling because of a lack of awareness of such services within the wider community. It was also argued that such cultural barriers should be taken into account in order to understand better the lived-experiences of a community. Group sessions, or the facets of the interactions at school that successfully facilitated self-expression, could function as a safe refuge for students and ultimately develop a sense of community. Finally, in relation to such a feeling of community, future researchers can conduct similar studies in subcultural contexts (e.g., popular culture).

\section{Acknowledgements}

The author would like to thank Ken Wilson, Psy.D., for his thoughtful supervision, as well as Junko Ishikawa (M.A. in Applied Theatre) for her helpful comments on conscientization. The author also wishes to express great appreciation to Mark E. Koltko-Rivera, Ph.D., for his guidance and encouragement. The author, however, bears full responsibility for this article.

\section{Conflicts of Interest}

The author declares no conflicts of interest regarding the publication of this paper.

\section{References}

Anderson, A. R., Christenson, S. L., Sinclair, M. F., \& Lehr, C. A. (2004). Check \& Connect: The Importance of Relationships for Promoting Engagement with School. Journal of School Psychology, 42, 95-113. https://doi.org/10.1016/j.jsp.2004.01.002

Bakhtin, M. M. (1981). The Dialogic Imagination: Four Essays. Austin, TX: University of Texas Press.

Dalton, J., Elias, M., \& Wandersman, A. (2001). Community Psychology: Linking Individuals and Communities (2nd ed.). Belmont, CA: Thomson Wadsworth.

Ellis, C., Adams, T. E., \& Bochner, A. P. (2011). Autoethnography: An Overview. Forum: Qualitative Social Research, 12, Article No. 10. http://nbn-resolving.de/urn:nbn:de:0114-fqs1101108

Esteban-Guitart, M. (2014). Appropriation. In T. Teo (Ed.), Encyclopedia of Critical Psychology. New York: Springer. https://doi.org/10.1007/978-1-4614-5583-7_616 https://link.springer.com/referenceworkentry/10.1007/978-1-4614-5583-7_616

Freire, P. (2000). Pedagogy of the Oppressed (30th Anniversary ed.). New York: Continuum.

Gannon, S. (2017). Autoethnography. In Oxford Research Encyclopedia of Education (pp. 1-21). Oxford: Oxford University Press. https://doi.org/10.1093/acrefore/9780190264093.013.71

Gergen, K. (1985). The Social Constructionist Movement in Modern Psychology. American Psychologist, 40, 266-275. https://doi.org/10.1037/0003-066X.40.3.266

Grbich, C. (2012). Qualitative Data Analysis: An Introduction (2nd ed.). London: Sage. 
Hess, A. K. (2009). Sense-of-the-Other: At the Core of Interpersonal Theory and Practice. Journal of Contemporary Psychotherapy, 39, 25-32.

https://doi.org/10.1007/s10879-008-9098-9

Hidalgo, M. C., \& Hernández, B. (2001). Place Attachment: Conceptual and Empirical Questions. Journal of Environmental Psychology, 21, 273-281. https://doi.org/10.1006/jevp.2001.0221

Kazdin, A. E., \& Weisz, J. R. (2003). Evidence-Based Psychotherapies for Children and Adolescents. New York: Guilford Publications.

Levenson, E. A. (2005). The Fallacy of Understanding: An Inquiry into the Changing Structure of Psychoanalysis; the Ambiguity of Change: An Inquiry into the Nature of Psychoanalytic Reality. Hillsdale, NJ: The Analytic Press.

Mateu-Gelabert, P., \& Lune, H. (2007). Street Codes in High School: School as an Educational Deterrent. City \& Community, 6, 173-191. https://doi.org/10.1111/j.1540-6040.2007.00212.x

Sarason, S. B. (1974). The Psychological Sense of Community: Prospects for a Community Psychology. Cambridge, MA: Brookline Books.

Sullivan, H. S. (1953). The Interpersonal Theory of Psychiatry. New York: W. W. Norton.

Vontress, C. E. (1969). Cultural Barriers in the Counseling Relationship. Personnel \& Guidance Journal, 48, 11-17. https://doi.org/10.1002/j.2164-4918.1969.tb03276.x

Vontress, C. E. (1974). Barriers in Cross-Cultural Counseling. Counseling \& Values, 18, 160-165. https://doi.org/10.1002/j.2161-007X.1974.tb01065.x

Walrond-Skinner, S. (1986). Interpersonal School (of Psychoanalysis). In Dictionary of Psychotherapy (pp. 187-188). New York: Routledge \& Kegan Paul.

Yancy, A. L. (1984). Crossing the Barrier of Counseling Minority Students. Journal of College Student Personnel, 25, 172-173. 